\section{Inherited cardiac disease}

Perry Elliott, Pier D. Lambiase, Dhavendra Kumar

Oxford specialist handbook in Cardiology, Oxford University Press; 2011.

ISBN: 978-0-19-955968-8

Pages 405; £39.99

Major advances have been achieved over the two last decades in the field of genetic cardiovascular diseases, not only through increased recognition and understanding of underlying molecular defects but also through rapid translation of knowledge into clinical practice. Genetic counseling and organization of cardiac family screening has become part of the medical management of these diseases, and these should be performed systematically unless an acquired cause has been diagnosed. At the same time, genetic testing was rapidly developed and can be discussed in various situations, including diagnostic purpose, predictive diagnosis in family members, risk stratification and prenatal diagnosis. Moreover, appropriate application of the growing knowledge in clinical practice has been underlined as a clear goal in recent guidelines from several national and international societies. In this context, this book is more than welcome in helping the clinician achieve these goals.

The book was written by 17 contributors from the UK and is organized into 20 chapters. After the introduction (chapter 1), chapters 2 to 5 deal with general principles of genetics (medical genetics, laboratory techniques, genetic counseling, genetic testing and screening). The various inherited cardiac conditions are described in chapters 6 to 19 that describe congenital heart diseases, chromosomal disorders, cardiomyopathies, inherited arrhythmias and conduction disorders, connective tissue disorders, familial hypercholesterolemia, coronary artery disease, stroke, pulmonary arterial hypertension, hereditary hemorrhagic telangiectasia, inherited skeletal muscle disease, mitochondrial diseases, inherited metabolic diseases and inherited hematologic disorders. Finally, chapter 20 is related to pharmacogenetics.

The style of presentation of each chapter is very concise and the authors have managed to strike a balance between providing key information while keeping the size of the chapters appropriate for a handbook. This is really a book you would like to have in your pocket and open regularly during the day. Chapters contain few figures or tables and concise selected references. Some sections of the chapters are particularly welcome as their content is very helpful in the diagnosis (e.g. interpretation of the laboratory report in chapter 3 , principles of genetic counseling in chapter 4) or management (e.g. differential diagnosis of hypertrophic cardiomyopathy, therapeutic management of long QT and Brugada syndrome) of the condition.

Given the format of the book, it is not surprising that some sections appear rather too brief, such as that on congenital heart diseases. Figures about the phenotype would also have been welcome as key diagnostic clues to illustrate some chromosomal disorders, metabolic or mitochondrial diseases. Therapeutic management is quite heterogeneous in the various chapters and sometimes poorly described, as for arrhythmogenic right ventricular cardiomyopathy. A few sections suffer from excessive approximations, such as the definition of polymorphisms (page 8) (in fact they can sometimes lead to altered protein function) or genetic testing, the title and content of chapter 5 , that is in fact dedicated to predictive testing, or pre-symptomatic testing in family members. Some references are lacking (European or US guidelines about genetic testing) and should be added in any future edition of the book. Finally, some molecular strategies that are described are more related to research than medical practice (such as linkage analyses) but recent new technologies that are entering the clinical arena are not described (e.g. next generation sequencing).

Taken as a whole, the quality of the book is obvious and it will provide a lot of important information for the clinicians dealing with inherited cardiac diseases, be they student or practising physician, cardiologist or geneticist. I would strongly recommend this book.

Philippe Charron, MD, PhD. Referral center for inherited cardiac diseases, UPMC University Paris 6, Hôpital PitiéSalpêtrière, Paris, France 